\title{
Spectral Efficiency Improvements in HetNets by Exploiting Device-to-Device Communications
}

\author{
Hafiz A. Mustafa, Muhammad Z. Shakir, Yusuf A. Sambo, Khalid A. Qaraqe, \\ Muhammad A. Imran, and Erchin Serpedin
}

\begin{abstract}
Next generation cellular networks require huge capacity, ubiquitous coverage and maximum energy efficiency. In order to meet these targets, Device-to-device (D2D) communication is being considered for future heterogeneous networks (HetNets). In this paper, we consider a three tier hierarchical HetNet by exploiting D2D communication in traditional HetNet. D2D communication is deployed within the HetNet where closely located mobile users are engaged in direct communication without routing the traffic through cellular access network. The proposed configuration mandates to reduce the interference offered by the resultant HetNet by reducing the transmitter-receiver distance and ensuring that the mobile users are transmitting with adaptive power subject to maintaining their desired link quality. In this context, we analyzed and compared the spectral efficiency improvements in hierarchical HetNet against traditional HetNet. Simulation results show that D2D communication offers much higher spectral efficiency as compared to traditional HetNet.
\end{abstract}

Index Terms-Hierarchical HetNet, D2D communication, small base stations, spectral efficiency, power control.

\section{INTRODUCTION}

C APACITY and coverage enhancement has been the constant goal of every wireless communication system. With the advent of mobile data services and smart devices, the capacity requirements exploded in recent years with worldwide mobile traffic forecast of more than 127 exabyte (EB) in 2020 [1]. An increase of thousand-fold in wireless traffic is expected in 2020 as compared to 2010 figures with expected figure of 50 billion communication devices [2]. This explosive growth of mobile traffic can be handled by capacity enhancement which mainly comprises of three techniques: spectral efficiency, spectral aggregation, and network densification [3]. The spectral efficiency approaches mainly target interferenceaware and cooperative communication e.g. coordinated beamforming, multiple-input multiple-output (MIMO), coordinated multi-point (CoMP), and Device-to-device cooperation. The spectrum aggregation comprises of carrier aggregation to enhance system bandwidth.

H. A. Mustafa, Y. Sambo, and M. A. Imran are with Center for Communication Systems Research (CCSR), University of Surrey, Guildford, UK. Emails: \{h.mustafa, yusuf.sambo,m.imran\}@surrey.ac.uk

M. Z. Shakir, and K. A. Qaraqe are with Electrical and Computer Engineering Dept., Texas A\&M University at Qatar, Doha. Emails: \{muhammad.shakir, khalid.qaraqe\}@qatar.tamu.edu

E. Serpedin is with Electrical and Computer Engineering Dept., Texas A\&M University, College Station, TX. Email: serpedin@ece.tamu.edu

This publication was made possible by NPRP grant 4-1293-2-513 from the Qatar National Research Fund (a member of Qatar Foundation). The statements made herein are solely the responsibility of the authors.
The network densification has been globally accepted as the quick and cost-effective solution to meet capacity and coverage demands. The deployment of huge number of small cells has been noticed in the past [4] which results in heterogeneous networks (HetNets) where several types of low-power smallcell base stations (SBSs) such as femto-cell, pico-cell and relays, are deployed within a macro-cell BS (MBS) coverage area to improve the spectral efficiency and coverage of cellular networks. SBS deployments ensure better transmission quality due to the short distance between the small-cell users and the associated SBSs, thus, improving the network spectral efficiency (SE) [5, 6]. It has been shown in [7] that the deployment of pico-cells can improve user throughput and expand the range of cells. In [8], the authors proposed an efficient distribution of femto-cells within MBS based on the minimum allowable received signal power at the user. It was shown that cell coverage area was increased by twofold using the efficient femto-cell location deployment. The authors in [9] proposed a heterogeneous deployment of femtocells around the cell-edge of a macro-cell to improve the area spectral efficiency (ASE) of the network. On the other side, the SBS deployment in HetNets requires substantial infrastructure where the cellular traffic route through the SBS even in the situation where the communicating devices are close to each other $[10,11]$. Moreover, SBS deployment requires additional link to backhaul the traffic to the core cellular network which increases the capital and operational expenditures for the operators [12-14].

With the spectral performance of the wireless link approaching the theoretical limits due to present cellular wireless networks, researchers have been working on various aspects in the frame-work of LTE-Advanced to further facilitate the mobile users in a ubiquitous and cost effective manner. One of the ways of increasing the achievable rate in cellular communications is direct communication between closely located mobile users. This form of communication is referred to as device-to-device (D2D) communication [15, 16]. Mobile devices involved in D2D communication form a direct link with each other, without the need of routing traffic via the cellular access network, resulting in lower transmit power and end-to-end delay, as well as freeing network resources. The lower transmit powers manifest through reduced interference levels in the system and battery power savings, while the improved rate is achieved as a result of low path-loss between any pair of devices involved in D2D communication [17].

In this context, we propose a three-tier hierarchical HetNet 
where D2D communication is introduced as tier 3 network within MBS (tier 1) and SBS (tier 2) to improve the SE of the considered HetNet, such that percentage of the mobile users engages in D2D communication in both higher tiers. D2D communication signaling could either be carried out through the macrocell access network or Wi-Fi access points. This deployment setting is compared with traditional HetNet in terms of capacity enhancement.

The rest of this paper is organized as follows. Section II describes the system model including network architecture, spectrum partitioning and transmission model. Section III presents spectral analysis of traditional and hierarchical HetNet. Section IV presents the simulation results depicting capacity gains by exploiting D2D communication in hierarchical HetNets. Finally, Section V concludes the paper.

\section{SYSTEM MODEL}

In this section, we describe network architecture, spectrum partitioning and transmission model of hierarchical HetNet.

\section{A. Network Architecture}

The hierarchical HetNet comprises of the following tiers:

- Tier One: Macro-cell users connected to MBS.

- Tier Two: Small-cell users connected to the SBS.

- Tier Three: D2D users connected to MBS and SBSs.

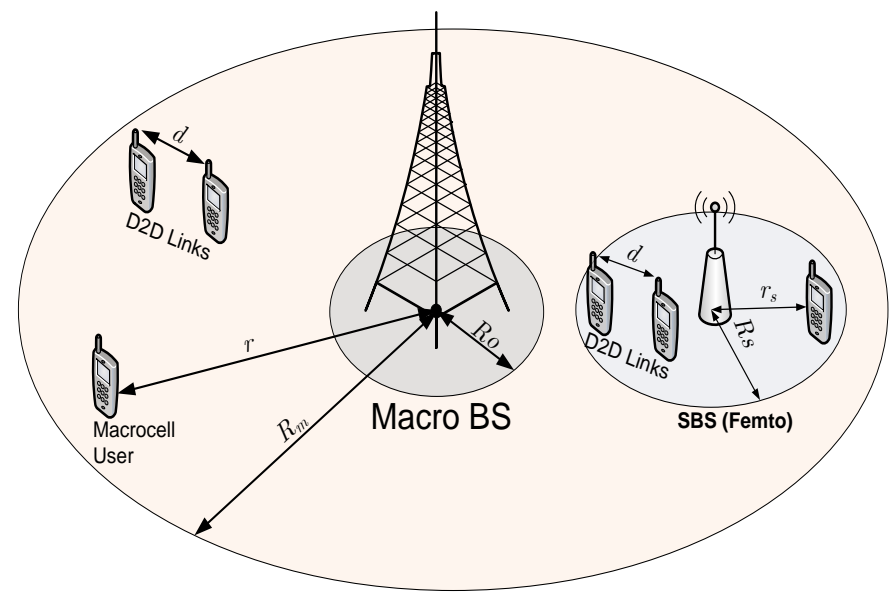

Fig. 1: Hierarchical heterogeneous network showing MBS, SBS, and D2D communication in both higher tiers.

The network shown in Fig. 1 contains $U=\mu_{m} \pi\left(R_{m}^{2}-R_{0}^{2}\right)$ users distributed between $R_{m}$ and $R_{0}$, where $R_{m}$ is the macro-cell radius, $R_{0}$ is the minimum distance between a mobile user and MBS, and $\mu_{m}$ is the user density per $\mathrm{m}^{2}$ in the coverage area of MBS. For the sake of simplicity, we have shown only one MBS in the top tier, however, we assume $Q-1=6$ interfering co-channel MBSs near the reference MBS. We consider UEs to be mutually independent and uniformly distributed throughout the network. Hence, the probability density function (PDF) of MBS users with polar coordinate $(r, \theta)$ is given by

$$
p(r)=\frac{2\left(r-R_{0}\right)}{\left(R_{m}-R_{0}\right)^{2}}, P(\theta)=\frac{1}{2 \pi},
$$

where $R_{0} \leq r \leq R_{m}$ and $0 \leq \theta \leq 2 \pi$.

Let $U_{m}=(1-\eta) U$ randomly distributed UEs are connected to MBS such that $\eta$ is the percentage of users that are offloaded to SBSs. According to [18], wireless usage is shifting indoors where the majority of mobile traffic, approximately $80 \%$ is indoor and nomadic, rather than truly mobile. In this paper, we assume $\eta$ to be $80 \%$ so that remaining $20 \%$ users are connected to MBS. Therefore, D2D communication in MBS and SBS is emerging as possible solution to address such modern mobile traffic patterns in HetNets.

Let $U_{m}^{d 2 d}=U_{m} \zeta_{m}^{d 2 d}$ is the number of MBS users involved in D2D communication where the parameter $0 \leq \zeta_{m}^{d 2 d} \leq 1$ is the content exchange information which describes the probability that the devices exploit the caching in MBS and share the content (peer-to-peer networking, single/multiple-hop relaying etc) and establish direct link over D2D protocol. The parameter $\zeta_{m}^{d 2 d}$ may be modeled probabilistically representing the usage of caching in MBS. Under such modeling, $U_{m}^{c u}=U_{m}(1-$ $\left.\zeta_{m}^{d 2 d}\right)$ gives number of MBS cellular users.

Let $U_{s}=\pi \mu_{s} R_{s}^{2}$ is the number of the users in each of the SBSs such that $\mu_{s}$ is the user density of $s^{\text {th }}$ SBS. The number of SBSs required to cover the MBS coverage area is

$$
N=\left\lceil\frac{U-U_{m}}{U_{s}}\right\rceil,
$$

where $\lceil x\rceil$ is the smallest integer not less than $x ; U_{s}$ is the number of users in any of the SBS.

Similar to MBS users, the PDF of SBS users with polar coordinates $\left(r_{s}, \theta_{s}\right)$, is given by

$$
p\left(r_{s}\right)=\frac{2 r_{s}}{R_{s}^{2}}, P\left(\theta_{s}\right)=\frac{1}{2 \pi},
$$

where $0 \leq r_{s} \leq R_{s}, 0 \leq \theta_{s} \leq 2 \pi$.

Let $\zeta_{s}^{d 2 d}$ denotes the content exchange information of $s^{t h}$ SBS where the corresponding users are involved in D2D communication for device centric and low-mobility indoor activities (gaming, ultra high definition video sharing etc). In this case, $U_{s}^{c u}=U_{s}\left(1-\zeta_{s}^{d 2 d}\right)$ gives number of SBS cellular users (not involved in D2D communications) whereas the total users involved in D2D communication in entire small-cell network can be calculated as

$$
U_{s}^{d 2 d}=\pi R_{s}^{2} \sum_{n=1}^{N} \mu_{s} \zeta_{s}^{d 2 d}
$$

such that $U_{s}^{d 2 d} / 2$ is the total number of D2D pairs in smallcell network. The remaining users of all SBSs, not involved in D2D communication, are given as

$$
U_{s}^{t c u}=U_{s} N-U_{s}^{d 2 d} .
$$

\section{B. Spectrum Partitioning}

We assume dedicated carrier deployment in the network, where the MBS, SBS and D2D communication users operate on separate bandwidths based on the active number of users 
associated with each technology. Let the total available spectrum be $w_{t}[\mathrm{~Hz}]$. It follows that for traditional HetNet

$$
w_{t}=w_{m}+w_{s},
$$

whereas for hierarchical HetNet

$$
w_{t}=\left(w_{m}^{H H}+w_{m}^{d}\right)+\left(w_{s}^{H H}+w_{s}^{d}\right),
$$

where $w_{m}=w_{t}\left(U_{m} / U\right)$ and $w_{s}=w_{t}\left(U_{n} N / U\right)$ are the dedicated channels of each MSB and SBS user in traditional HetNet respectively. Similarly, $w_{m}^{H H}=w_{t}\left(U_{m}^{c u} / U\right), w_{s}^{H H}=$ $w_{t}\left(U_{s}^{t c u} / U\right), w_{m}^{d}=w_{t}\left(U_{m}^{d 2 d} / U\right)$, and $w_{s}^{d}=w_{t}\left(U_{s}^{d 2 d} / U\right)$ are the dedicated channels of each MBS, SBS and D2D user in hierarchical HetNet respectively. The number of channels in both MBS and SBSs are assumed to be equal to the number of users they contain and each channel is allocated to a single user [19]. Hence, the interference received at the MBS or SBS is from the mobile users in each of the neighboring cochannel macro/small-cells that are transmitting on the same channel while interference in each D2D communication link is assumed to be from the closest D2D communication user that is not part of that communication link. This assumption was made because mobile devices engaged in D2D communication usually transmit with very low power which causes reduced interference.

\section{HetNet Transmission Design}

The received signal power at a distance $r$ for MBS, SBSs and one of the terminal of devices which are engaged in D2D communication is given by

$$
P^{r x}=P^{t x} r^{-\alpha}(1+r / g)^{-\beta} \Gamma,
$$

where $\alpha$ and $\beta$ stand for basic and additional path-loss exponents, respectively and $\Gamma$ denotes a path-loss dependent constant. The parameter $g=\frac{4 H_{b s} H_{u}}{\lambda_{c}}[\mathrm{~m}]$ is the breakpoint of the path-loss curve such that $H_{b s}$ [m] represents the BS antenna height, $H_{u}[\mathrm{~m}]$ denotes the mobile user antenna height and $\lambda_{c}[\mathrm{~m}]$ stands for the wavelength of the carrier frequency, $\mathrm{F}_{c}{ }^{1} . P^{t x}[\mathrm{~W}]$ defines the mobile user uplink transmit power such that all users in the considered configurations of HetNet are assumed to transmit with closed loop power control mechanism while maintaining a certain received signal threshold ${ }^{2}$ [20]. The adaptive transmit power is based on two-slope pathloss model [21] given by

$$
P^{t x}[\mathrm{~W}]=\min \left(P_{\max }, P_{0}\left(10^{P L(r) / 10}\right)\right),
$$

where

$P L(r)[\mathrm{dB}]=10 \log _{10} r^{\alpha}+10 \log _{10}(1+r / g)^{\beta}-10 \log _{10} \Gamma$,

\footnotetext{
${ }^{1}$ The breakpoint of the path-loss curve is the point after which the strength of the signal attenuates such that the basic path-loss exponent $\alpha$ represents the slope before breakpoint and $\alpha+\beta$ represents the slope after breakpoint.

${ }^{2}$ Closed loop power control is the ability of the mobile user to adapt its uplink transmit power in accordance with one or more Transmit Power Control (TPC) commands received in the downlink from their respective BS.
}

is the path-loss of a mobile user, $P_{\max }[\mathrm{W}]$ is the maximum uplink transmit power of a mobile device, $P_{0}[\mathrm{~W}]$ is the received signal power threshold. For D2D communication, $H_{b s}=H_{u}$ because the heights of the mobile users are assumed to be equal, the path-loss exponents are the same with those of the small-cells and $r=d$ in (1) and (2). Substituting (2) into (1), the received signal power can be expressed as

$$
P^{r x}[\mathrm{~W}]= \begin{cases}\frac{P_{\max }}{10^{P L(r) / 10}} & P_{\max }<P_{0} 10^{P L(r) / 10} \\ P_{0} & \text { otherwise }\end{cases}
$$

\section{Spectral EFFiciency AnAlysis}

In this section, we present spectral analysis of traditional and hierarchical HetNets.

\section{A. Traditional HetNet}

The sum rate of traditional HetNet (without D2D communication) comprises of individual sum rates of MBS and SBSs as

$$
C=C_{m}+C_{s}=\sum_{l=1}^{U_{m}} C_{l, m}+\sum_{s=1}^{N} \sum_{z=1}^{U_{s}} C_{z, s},
$$

where $C_{m}$ [bits/s] is the sum-rate of MBS and $C_{s}$ [bits/s] is the sum-rate of SBSs. The achievable capacity $C_{l, m}$ of the $l^{t h}$ user located in $m^{\text {th }}$ MBS of traditional HetNet is given by

$$
\begin{aligned}
C_{l, m} & =w_{m} \mathbb{E}\left[\log _{2}\left(1+\gamma_{l, m}\right)\right], \\
& =w_{m} \int_{0}^{\infty} \log _{2}\left(1+\gamma_{l, m}\right) f_{\gamma}\left(\gamma_{l, m}\right) d \gamma_{l, m},
\end{aligned}
$$

where $f_{\gamma}\left(\gamma_{l, m}\right)$ denotes the PDF of $\gamma_{l, m}$, and $\gamma_{l, m}$ is the signal to interference ratio (SIR) of the desired link. Assuming the thermal noise power is negligible compared to the co-channel interference power, the SIR of the $l^{t h}$ user located in $m^{t h}$ macro-cell can be expressed as

$$
\gamma_{l, m}=\frac{P_{l, m}^{r x}}{\sum_{\tau=1, \tau \neq m}^{Q} P_{l, \tau}^{r x}},
$$

where $P_{l, m}^{r x}[\mathrm{~W}]$ is the received power at the $m^{t h}$ macrocell from the $l^{\text {th }}$ user and $\sum_{\tau=1, \tau \neq m}^{Q} P_{l, \tau}^{r x}$ is the sum of the individual interfering power levels received at the reference MBS from the interfering mobile users $\left\{l_{\tau}\right\}_{\tau=1, \tau \neq m}^{Q}$ which are located in each of the $Q-1=6$ interfering MBSs. Substituting (1) into $^{3}(6)$, SIR of macro user is given by

$$
\gamma_{l, m}=\frac{P_{l, m}^{t x} r_{l, m}^{-\alpha_{m}}\left(g_{m}+r_{l, m}\right)^{-\beta_{m}}}{\sum_{\tau=1, \tau \neq m}^{Q} P_{l, \tau}^{t x} r_{l, \tau}^{-\alpha_{m}}\left(g_{m}+r_{l, \tau}\right)^{-\beta_{m}}},
$$

Similarly $C_{z, s}$ is the achievable capacity of the $z^{t h}$ user in $s^{\text {th }}$ small cell, and is given by

$$
\begin{aligned}
C_{z, s} & =w_{s} \mathbb{E}\left[\log _{2}\left(1+\gamma_{z, s}\right)\right], \\
& =w_{s} \int_{0}^{\infty} \log _{2}\left(1+\gamma_{z, s}\right) f_{\gamma}\left(\gamma_{z, s}\right) d \gamma_{z, s},
\end{aligned}
$$

\footnotetext{
${ }^{3}$ In (1), we suppress the notations for sake of simplicity and better understanding.
} 
such that $f_{\gamma}\left(\gamma_{z, s}\right)$ is the PDF of $\gamma_{z, s}$ and $\gamma_{z, s}$ is the SIR of the $z^{\text {th }}$ user in $s^{t h}$ small cell which is expressed as

$$
\gamma_{z, s}=\frac{P_{z, s}^{r x}}{\sum_{v=1, v \neq s}^{N} P_{z, v}^{r x}} .
$$

The parameter $P_{z, s}^{r x}[\mathrm{~W}]$ in (9) represents the received power at the $s^{t h}$ small BS from the $z^{t h}$ user and $\sum_{v=1, v \neq s}^{N} P_{z, v}^{r x}$ is the sum of the power received at the $s^{\text {th }}$ small BS from the interfering small cell users $\left\{z_{v}\right\}_{v=1, v \neq s}^{N}$ in the neighboring $N-1$ interfering small BSs in HetNet. Substituting (1) into ${ }^{2}$ (9), the SIR of the small cell user is expressed as

$$
\gamma_{z, s}=\frac{P_{z, s}^{t x} r_{z, s}^{-\alpha_{s}}\left(g_{s}+r_{z, s}\right)^{-\beta_{s}}}{\sum_{v=1, v \neq s}^{N} P_{z, v}^{t x} r_{z, v}^{-\alpha_{s}}\left(g_{s}+r_{z, v}\right)^{-\beta_{s}}} .
$$

\section{B. Hierarchical HetNet}

The capacity of hierarchical HetNet consists of cellular and D2D users in both MBS and SBSs. In case of MBS, we have $U_{m}^{c u}$ cellular and $U_{m}^{d 2 d}$ D2D users whereas for each SBS, we have $U_{s}^{c u}$ cellular and $U_{s}^{d 2 d}$ D2D users. The total capacity [bits/s] of hierarchical HetNet can be expressed as

$$
\begin{aligned}
C^{H H} & =C_{m}^{H H}+C_{s}^{H H} \\
& =\sum_{l=1}^{U_{m}^{c u}} C_{l, m}^{H H}+\sum_{x=1}^{U_{m}^{d 2 d}} C_{x, m}^{H H} \\
& +\sum_{s=1}^{N}\left[\sum_{z=1}^{U_{s}^{c u}} C_{z, s}^{H H}+\sum_{x=1}^{U_{s}^{d 2 d}} C_{x, s}^{H H}\right],
\end{aligned}
$$

where $C_{m}^{H H}$ comprises of capacity of $U_{m}^{c u}$ cellular and $U_{m}^{d 2 d}$ D2D users of MBS. Similarly the capacity $C_{s}^{H H}$ is the capacity of $U_{s}^{c u}$ cellular and $U_{s}^{d 2 d}$ D2D users of each SBS. $C_{l, m}^{H H}$ and $C_{z, s}^{H H}$ represent achievable capacity of MBS and SBS cellular users calculated similar to (5) and (8), respectively.

The achievable capacity of the $x^{\text {th }}$ D2D communication user in MBS or SBS is expressed as

$$
\begin{aligned}
C_{x, y}^{H H} & =w_{y}^{d} \mathbb{E}\left[\log _{2}\left(1+\gamma_{x}\right)\right], \\
& =w_{y}^{d} \int_{0}^{\infty} \log _{2}\left(1+\gamma_{x}\right) f_{\gamma}\left(\gamma_{x}\right) d \gamma_{x},
\end{aligned}
$$

for $y \in\{m, s\}$. The $f_{\gamma}\left(\gamma_{x}\right)$ denotes PDF of the desired SIR $\gamma_{x}$ of $x^{t h}$ D2D communication user in MBS or SBS which is given by

$$
\gamma_{x}=\frac{P_{x, y}^{r x}}{P_{x, i}^{r x}}
$$

where $P_{x, y}^{r x}$ is the $x^{t h}$ D2D user's received power at its D2D partner in MBS or SBS and $P_{x, i}^{r x}$ is the received interference power at the $x^{t h}$ D2D user from the interfering D2D user $i$.

Substituting (1) into (13), the SIR of the $x^{\text {th }}$ mobile user for $y \in\{m, s\}$ is expressed as

$$
\gamma_{x}=\frac{P_{x, y}^{t x} d_{x, y}^{-\alpha_{d}}\left(g_{d}+d_{x, y}\right)^{-\beta_{d}}}{P_{i, y}^{t x} d_{i, y}^{-\alpha_{d}}\left(g_{d}+d_{i, y}\right)^{-\beta_{d}}} .
$$

\section{NumericAl Results}

In this section, we evaluate the performance gains of traditional and hierarchical HetNet in terms of variable user density. To this end, we performed extensive monte-carlo simulations assuming system parameters summarized in Table I. An ultra

Table I: Simulation Parameters

\begin{tabular}{|c|c||c|c|}
\hline Parameter & Value & Parameter & Value \\
\hline$R_{m}[\mathrm{~m}]$ & 560 & $R_{s}[\mathrm{~m}]$ & 30 \\
\hline$P_{\max }[\mathrm{W}]$ & 0.8 & $P_{0}[\mu \mathrm{W}]$ & 0.8 \\
\hline$\alpha_{m}=\beta_{m}$ & 2.1 & $\alpha_{s}=\beta_{s}$ & 1.8 \\
\hline$\alpha_{d}=\beta_{d}$ & 1.8 & $\mu_{m}$ & $0.001-0.02$ \\
\hline$H_{b s}($ macro) $[\mathrm{m}]$ & 25 & $H_{b s}(\mathrm{SC})[\mathrm{m}]$ & 5 \\
\hline$H_{u}[\mathrm{~m}]$ & 2 & $\Gamma$ & 1 \\
\hline$R_{0}[\mathrm{~m}]$ & 10 & $\lambda_{c}[\mathrm{~m}]$ & 0.125 \\
\hline$w_{t}[\mathrm{MHz}]$ & 20 & $\mathrm{~F}_{c}[\mathrm{GHz}]$ & 2.4 \\
\hline
\end{tabular}

dense environment is simulated by increasing the user density from 1 to 20 users $/ \mathrm{km}^{2}$. In order to deploy SBSs uniformly into the coverage area of MBS, the whole disc of radius $R_{m}$ is divided into circular rings. For illustrative purpose, few rings of hierarchical HetNet showing three-tier network are shown in Fig. 2. In such a hierarchical network, the whole

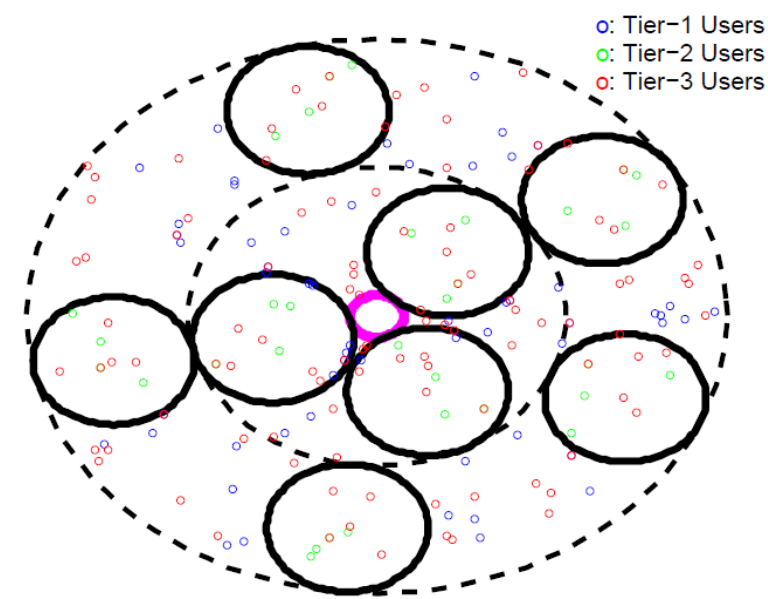

Fig. 2: Three-tier hierarchical HetNet showing only two-rings for illustrative purpose.

area is covered by the MBS with black circles showing SBS deployment. The small blue and green circles show MBS and SBS users directly connected to the respective BSs. The red small circles show D2D users either in MBS or SBSs as tier-3 network.

The probability of users for D2D communication depends on many factors including channel conditions, common contents etc. In order to choose D2D pairs in MBS $\left(\zeta_{m}^{d 2 d}\right)$ and SBS $\left(\zeta_{s}^{d 2 d}\right)$, the cumulative distribution function (CDF) for $U_{m}^{d 2 d}$ MBS and $U_{s}^{d 2 d}$ SBS users (distributed uniformly with the corresponding radii given in Table I) is approximated as shown in Fig. 3. Since MBS users are non-nomadic and fast moving as compared to SBS users, therefore the value of 

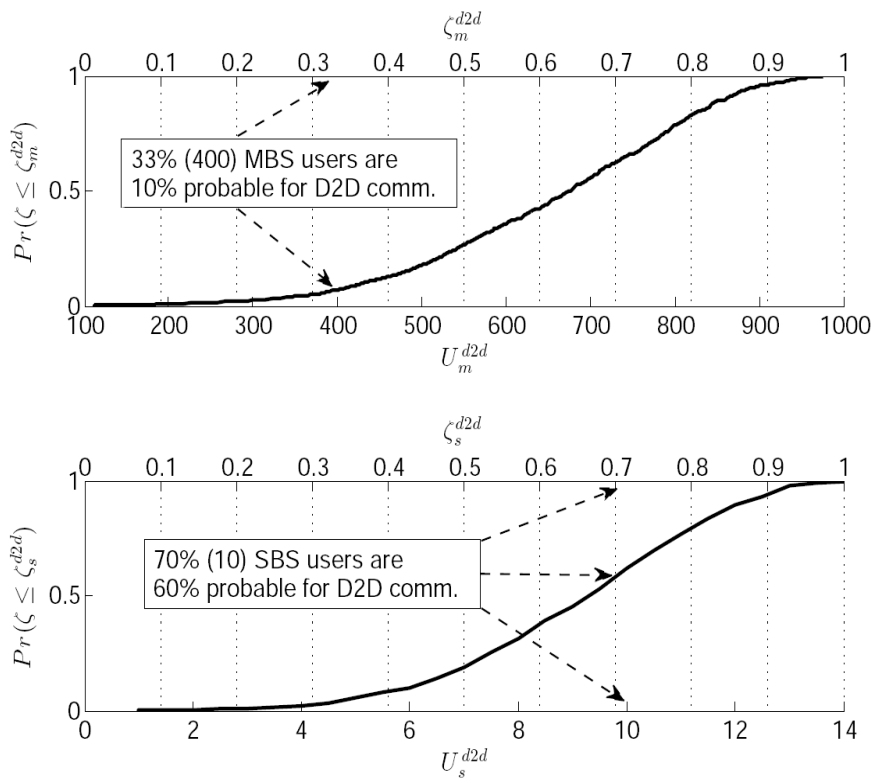

Fig. 3: D2D user density based on CDF approximation of $\zeta$.

$\zeta_{m}^{d 2 d} \sim 0.33$ is chosen which shows $10 \%$ proabability for D2D communication. For SBS users, the value of $\zeta_{s}^{d 2 d} \sim 0.70$ shows $60 \%$ probability for D2D users in small-cell. For illustrative purpose, the value of $\mu_{m}=0.005$ for $R_{m}=560 \mathrm{~m}$ and $R_{s}=30 \mathrm{~m}$, has been choosen which generates approximately 5000 users amongst which 1000 (20\%) are MBS and rest $(80 \%)$ are SBS users. In Fig. 3, the value of $\zeta_{m}^{d 2 d}$ corresponds to approximately $400 \mathrm{MBS}$ D2D users whereas $\zeta_{s}^{d 2 d}$ corresponds to approximately $10 \mathrm{D} 2 \mathrm{D}$ users per SBS.

The capacity enhancement of hierarchical HetNet is compared with the traditional HetNet in Fig. 4 and Fig. 5 where Fig. 4 shows the sum rate $(\mathrm{bps} / \mathrm{Hz})$ vs. variable user density for MBS and SBSs for the two cases of non-D2D and D2D users. The sum rate capacity increases with increased number of D2D users in hierarchical HetNet due to the frequency reuse whereas traditional HetNet shows constant sum rate. By increasing the users in traditional HetNet, the channel bandwidth per user reduces to accommodate the new users equally and fairly. However, the sum rate calculated for the increased number of users under fixed system bandwidth will remain constant as validated in simulation results. For hierarchical HetNet, the channel bandwidth for cellular user also reduces but D2D communication reuses channel bandwidth and hence results in sum rate enhancement. An interesting cross-over point is observed at 11 users $/ \mathrm{km}^{2}$ where single MBS with D2D links shows higher capacity as compared to huge deployments of SBSs with non-D2D links. This crossover point can be reached at low user density if the number of D2D links are increased further. However, D2D pairs can be exploited opportunistically depending on different factors e.g., shortest distance, channel conditions, common content information etc.

The overall system gain of hierarchical HetNet in Fig. 5 shows significant capacity enhancements as compared to constant sum rate of traditional HetNet. These capacity gains can further be enhanced by using non-orthogonal spectrum sharing and smart interference management techniques. In such a scenario, the optimum number of D2D pairs can be found, for example, by acheiving target SIR at desired node (cellular or D2D).

In Fig. 6, the interference geometry is drawn for traditional and hierarchical HetNet. Two user densities are simulated where first considers 1 user $/ \mathrm{km}^{2}$ and second considers 10 users $/ \mathrm{km}^{2}$ (closer to the cross-over point). In both cases, the CDF plot shows significant improvements in terms of required SIR and outage probability. For example to ensure outage probability of $10 \%$ in case of $1 \mathrm{user} / \mathrm{km}^{2}$, the HetNet with D2D links requires $26.66 \mathrm{~dB}$ less SIR as compared to traditional HetNet. Similarly, in case of 10 users $/ \mathrm{km}^{2}$, the SIR gain of $\gamma$ $=32.74 \mathrm{~dB}$ has been observed.

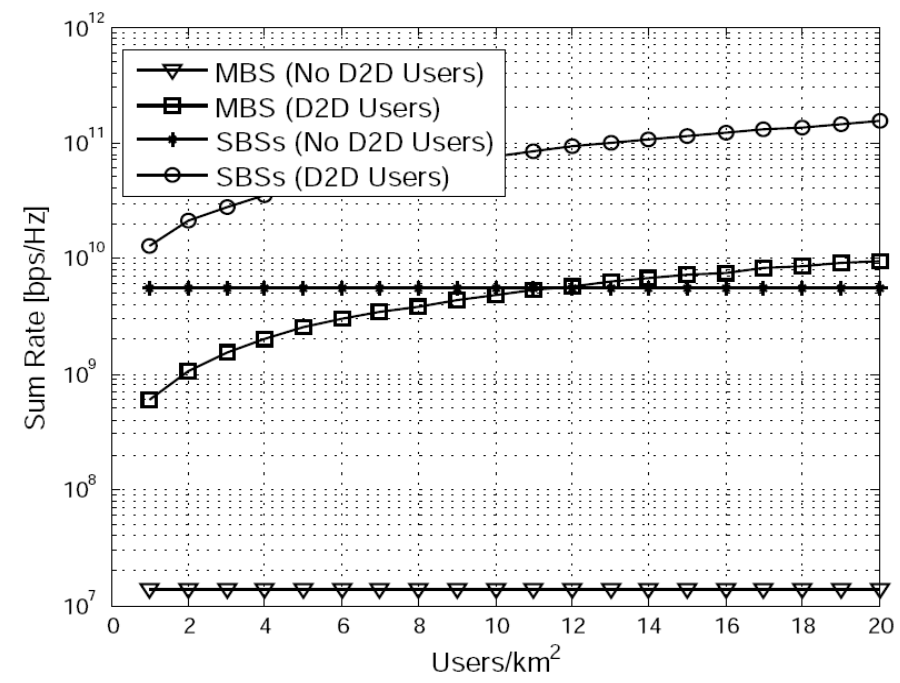

Fig. 4: Sum Rate of MBS, SBSs with/without D2D users.

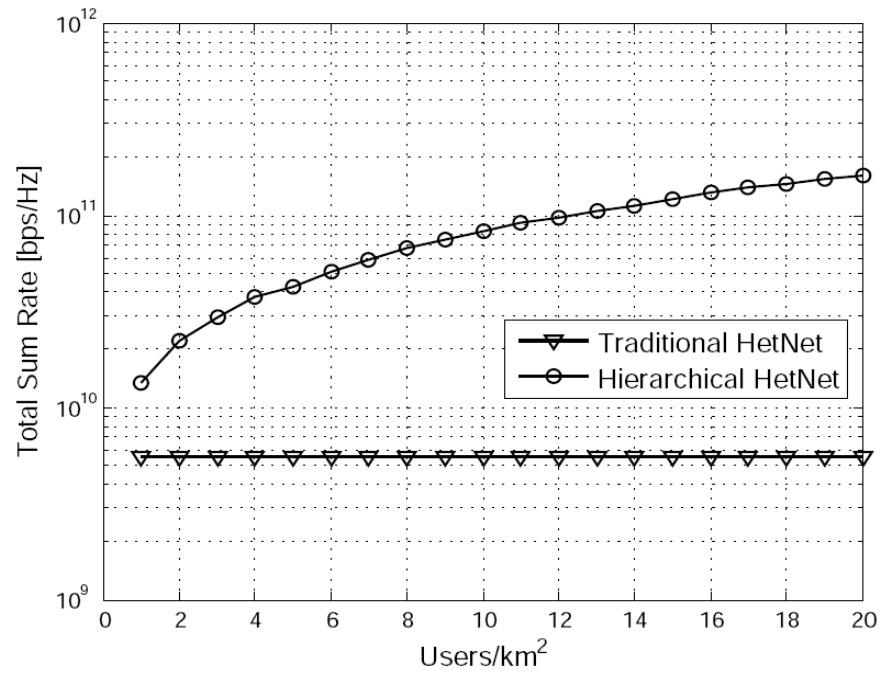

Fig. 5: Total Sum Rate of HetNet and hierarchical HetNet.

\section{Conclusions}

In this paper, we introduced a three-tier network as hierarchical HetNet in which D2D links are established in 


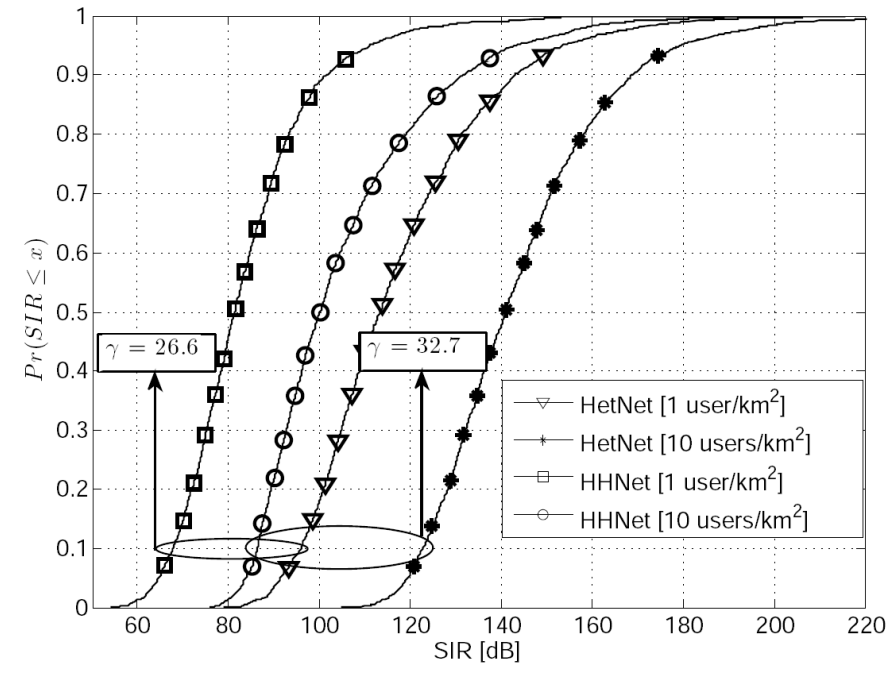

Fig. 6: Interference Geometry for two user densities

macro/small-cells. Two scenarios are simulated where the first comprised of HetNet without D2D links and the second considered hierarchical HetNet with overlay D2D communication. The capacity enhancements has been investigated by comparing the traditional HetNet with hierarchical HetNet. The D2D user density is varied from low to high values in order to simulate an ultra dense urban environment. Simulation results show that significant capacity enhancements can be achieved by exploiting D2D links in dense urban environment.

\section{REFERENCES}

[1] UMTS Forum, "UMTS Forum Report: Mobile Traffic Forecasts: 2010-2020," Report 44, January 2011.

[2] Ericsson, "More than 50 billion connected devices," White Paper, February 2011.

[3] H. Ishii, Y. Kishiyama, and H. Takahashi, "A novel architecture for LTE-B :C-plane/U-plane split and Phantom Cell concept," in Globecom Workshops (GC Wkshps), 2012 IEEE, Dec 2012, pp. 624-630.

[4] J. Andrews, H. Claussen, M. Dohler, S. Rangan, and M. Reed, "Femtocells: Past, Present, and Future," Selected Areas in Communications, IEEE Journal on, vol. 30, no. 3, pp. 497-508, April 2012.

[5] A. Damnjanovic, J. Montojo, W. Yongbin, J. Tingfang, L. Tao, M. Vajapeyam, Y. Taesang, S. Osok, and D. Malladi, "A survey on 3GPP heterogeneous networks," in IEEE Mag. Wireless Communs., vol. 18, no. 3, pp. 1021, Jun. 2011.

[6] I. Guvenc, "Capacity and fairness analysis of heterogeneous networks with range expansion and interference coordination," in IEEE Communs. Letters, vol. 15, no. 10, pp. 1084-1087, 2011.

[7] K. Okino, T. Nakayama, C. Yamazaki, H. Sato, and Y. Kusano, "Pico cell range expansion with interference mitigation toward LTE-Advanced heterogeneous networks," pp. 1-5, Jun. 2011.

[8] I. Shgluof, M. Ismail, and R. Nordin, "Efficient femtocell deployment under macrocell coverage in LTE-
Advanced system," in Proc. International Conference on Computing, Management and Telecommunications (ComManTel), 2013, Ho Chi Minh City, Vietnam, Jan. 2013, pp. 60-65.

[9] M. Z. Shakir and M. Alouini, "On the area spectral efficiency improvement of heterogeneous network by exploiting the integration of macro-femto cellular networks," in Proc. IEEE Intl. Conf. Communs., (ICC'12), pp. 1-6, Jun. 2012.

[10] Informa Telecoms \& Media, "Small Cell Market Status," Report for Small Cell Forum, no. 2, pp. 1-14, Jun. 2012.

[11] D. Calin, H. Claussen, and H. Uzunalioglu, "On femto deployment architectures and macrocell offloading benefits in joint macro-femto deployments," in IEEE Mag. Communs., vol. 48, no. 1, pp. 26-32, Jan. 2010.

[12] M. Z. Shakir, K. A. Qaraqe, H. Tabassum, M. S. Alouini, E. Serpedin, and M. A. Imran, "Green heterogeneous small-cell networks: toward reducing the $\mathrm{CO} 2$ emissions of mobile communications industry using uplink power adaptation," in IEEE Commun. Mag., vol. 51, no. 6, pp. 52-61, 2013.

[13] O. Tipmongkolsilp, S. Zaghloul, and A. Jukan, "The evolution of cellular backhaul technologies: Current issues and future trends," in IEEE Commun. Surveys \& Tutorials., vol. 13, no. 1, pp. 97-113, 2011.

[14] M. Paolini, L. Hiley, and F. Rayal, "Small-cell backhaul: Industry trends and market overview," http://www.altobridge.com/wp-content/uploads/2013/06/ SenzaFili_SCBackhaul.pdf, 2013.

[15] K. Doppler, M. Rinne, C. Wijting, C. Ribeiro, and K. Hugl, "Device-to-device communication as an underlay to LTE-advanced networks," in IEEE Mag. Communs., vol. 27, no. 12, pp. 42-49, Dec. 2009.

[16] P. Janis, C.-H. Yu, K. Doppler, C. B. Ribeiro, C. Wijting, K. Hugl, O. Tirkkonen, and V. Koivunen, "Device-todevice communication underlying cellular communications systems," in Intl. Jour. Commun., Network and System Sciences, vol. 2, no. 3, pp. 169-178, 2009.

[17] S. Hakola, T. Chen, J. Lehtomäki, and T. Koskela, "Device-to-device (D2D) communication in cellular network - performance analysis of optimum and practical communication mode selection," pp. 1-6, Apr. 2010.

[18] CISCO Systems, "Cisco service provider Wi-Fi: A platform for business innovation and revenue generation," Solution Overview, pp. 1-12, Jun. 2012.

[19] H. Tabassum, M. Z. Shakir, and M. Alouini, "Area green efficiency (AGE) of two tier heterogeneous cellular networks," pp. 529- 534, Dec. 2012.

[20] 3GPP, "Evolved Universal Terrestrial Radio Access (EUTRA); Physical Layer Procedures," 3rd Generation Partnership Project (3GPP), TS 36.213, Sept. 2008.

[21] P. Harley, "Short distance attenuation measurements at $900 \mathrm{MHz}$ and $1.8 \mathrm{GHz}$ using low antenna heights for microcells," in IEEE Jour. Selected Areas Communs., vol. SAC-7, pp. 5-11, Jan. 1989. 